\title{
Crystallography online by the bilbao crystallographic server
}

Gemma De La Flor Martin ${ }^{1}$, Emre Tasci ${ }^{2}$, Luis Elcoro ${ }^{3}$, Samuel Vidal ${ }^{3}$, Gotzon Madariaga ${ }^{3}$, Juan Manuel Perez-Mato ${ }^{3}$, Mois I. Aroyo ${ }^{3}$

${ }^{1}$ Dept. Earth Sciences, University Hamburg, Hamburg, Germany, ${ }^{2}$ Dept. Physics Eng., Hacettepe University, Ankara, Turkey,

${ }^{3}$ Departamento de Física de la Materia Condensada, Universidad del País Vasco UPV/EHU, Leioa, Spain

E-mail: gemma.delaflor@uni-hamburg.de

The Bilbao Crystallographic Server (www.cryst.ehu.es) [1] is a free web site that grants access to specialized databases and tools for the resolution of different types of crystallographic problems. The server is built on a core of databases that contain crystallographic data of space groups, magnetic space groups, subperiodic groups, plane and point groups and their symmetry relations. Parallel to the crystallographic software we have developed specialized tools for the analysis of complex solid- state physics and structural chemistry problems. The programs available on the Bilbao Crystallographic Server aims to bring the potential of symmetry and group theory to those users who are not necessarily experts but want to benefit from it in their studies. The purpose of this contribution is to report on the current state of the server emphasizing on the recently developed computer tools. The utility of the available applications will be demonstrated by illustrative examples.

The tools on the Bilbao Crystallographic Server are organized in eleven different shells, one of which is dedicated to the study of infrared, Raman and hyper-Raman scattering processes at the Brillouin-zone center. In this section one can find very useful programs to calculate the selection rules of these processes and the polarization selection rules for different configurations of the scattering experiment. The available data on group-subgroup relations between point and space groups and the corresponding correlations between their irreducible representations permit the analysis of the behaviour of the phonon modes during a symmetry break. The structure-utilities toolbox offers basic computer tools for transformation between different structure descriptions as well as more complex applications necessary for the comparison of structures and to evaluate their similarity [2]. Another important section on the server is the so-called Solid State Theory Applications shell which contains different types of tools for phase-transition studies. In this section one can find programs to study the crystal-structure relationship between two phases and online tools for the evaluation of the pseudosymmetry of a given structure with respect to a supergroup of its space group. Recently, due to the interest in the study of materials with diperiodic symmetry (layer and multilayer), new databases and tools have been developed to study this type of systems. Several programs in the server provide access to the general positions, Wyckoff positions, maximal subgroups and Brillouinzone databases. This shell also contains a program that identifies, for a given space group, the full set of possible layer symmetries of periodic sections defined by their common normal vector and location within the unit cell. On the other hand, magnetic symmetry is a powerful tool that eases the description and resolution of magnetic structures, constituting a complement and an alternative to the commonly used representation analysis. On the server there is also a shell devoted to magnetic space groups and magnetic symmetry [4] for the study of magnetic structures.

The Bilbao Crystallographic Server is in constant improvement and development and it is open access which makes it easier to diffuse the databases and the programs to the scientific community.

[1] M. I Aroyo et al. (2011) Bulgarian Chemical Communications, 43(2), 183-197

[2] G. de la Flor et al. (2016) J. Appl. Cryst., 49, 653-664

[3] J. M. Perez-Mato et al. (2015), Ann. Rev. Mater. Res., 45, 43.1-13.32

Keywords: Bilbao Crystallographic Server, on-line tools, symmetry 